\title{
New Unitary Relations between the QCD sum rules
}

\author{
Altug Ozpineci ${ }^{1, a}$ and Valeri Zamiralove,b \\ ${ }^{1}$ Physics Department, Middle East Technical University, 06531 Ankara, Turkey \\ ${ }^{2}$ Skobeltsyn Institute of Nuclear Physics, Lomonosov Moscow State University, 119991 Moscow, Russia
}

\begin{abstract}
New unitary symmetry relations are obtained between the QCD sum rules for $\Sigma \Lambda$ transitions. Also for QCD SR's with baryon distribution amplitudes the unitary relations are constructed, proved in a simple quark model and found to be consistent with the existing calculations.
\end{abstract}

\section{Introduction}

QCD sum rules (QCD SR's) introduced by [1,2] have become one of the most important tools while studying baryon or meson couplings and the corresponding form factors with photons, mesons or baryons. At the same time, the complexity of the formulas used in calculations has increased in obviously non-linear manner. So the control of one's own and other authors calculations becomes a non-trivial problem. Recently, we have proposed simple relations between the quantities involving $\Sigma$ hyperons and those involving $\Lambda$ hyperons [3]. They are generalized easily to any $\Sigma$-like and $\Lambda$-like baryons containing heavy quarks. Our formulas seem to be useful and for example they were inserted into the codes by Lai Wang and Frank Lee while studying magnetic moments of the octet baryons [4].

It is interesting that also the transition $\Sigma-\Lambda$ quantities could be obtained from the corresponding quantities of the $\Sigma$ or $\Lambda$ baryons. We want here to propose one more relation to control the complex calculations of the transition $\Sigma-\Lambda$ quantities which has the merit that it does not involve other baryon quantities, neither of $\Sigma$ nor of $\Lambda$ baryons.

The formula is rather trivial for nonrelativistic quark model or simple unitary symmetry model. However, it shows us the way to overcome many difficulties in the framework of QCD sum rules, as we shall see later on. In the very tedious QCD sum rule calculations it proves to be extremely useful. Its applicability was demonstrated in [3] on the example of sum rules for magnetic moments of octet baryons. In this report we are interested with the $\Sigma-\Lambda$ transitions. In [3] they were related to the $\Sigma$ and $\Lambda$ quantities as (we write in terms of correlators [1,2])

$$
\Pi^{\Sigma^{0}(d \leftrightarrow s)}-\Pi^{\Sigma^{0}(u \leftrightarrow s)}=-\Pi^{\Lambda(d \leftrightarrow s)}+\Pi^{\Lambda(u \leftrightarrow s)}=\sqrt{3} \Pi^{\Lambda \Sigma^{0}},
$$

where $d \leftrightarrow s$ means interchange of the flavors $d$ and $s$. The main point of our report is that it can be formulated in some "auto-sufficient" way. Our new relation between the corresponding correlation functions reads as

$$
\Pi^{\Lambda \Sigma^{0}(u \leftrightarrow s)}+\Pi^{\Lambda \Sigma^{0}(d \leftrightarrow s)}=\Pi^{\Lambda \Sigma^{0}}
$$

\footnotetext{
a e-mail: ozpineci@metu.edu.tr

be-mail: zamir@depni.sinp.msu.ru
} 


\section{Master formulas for the octet}

As the main ingredient of the polarization operator is the matrix element of the T-production of the interpolating currents, we now put our attention to it. The corresponding formulas are often named as "master formulas"[4]. We begin with the master formula for the $\Sigma^{0}$ [4], [7]:

$$
\begin{gathered}
\Sigma^{0} \equiv\left\langle 0\left|T\left\{\eta^{\Sigma^{0}} \bar{\eta}^{\Sigma^{0}}\right\}\right| V\right\rangle= \\
=-\varepsilon_{a b c} \varepsilon_{d e f}\left\{S_{u}^{a d} \gamma_{5} C S_{s}^{b e} C \gamma_{5} S_{d}^{c f}+S_{d}^{a d} \gamma_{5} C S_{s}^{b e} C \gamma_{5} S_{u}^{c f}+\right. \\
S_{u}^{a d} \operatorname{Tr}\left[\gamma_{5} C S_{s}^{b e} C \gamma_{5} S_{d}^{c f}\right]+S_{d}^{a d} \operatorname{Tr}\left[\gamma_{5} C S_{s}^{b e} C \gamma_{5} S_{u}^{c f}\right]+\ldots
\end{gathered}
$$

where $V$ could be meson or photon. It is vacuum state for the mass sum rule (SR). $S$ is the quark propagator [1],[2]. We write for our purpose just the first line, as for us it is important that in every line the quark flavors index is of the form

$$
\Sigma^{0}=u s d+d s u
$$

Now, we construct $\Lambda$-quantity using the relation from [3]

$$
\begin{gathered}
\Lambda \equiv\left\langle 0\left|T\left\{\eta^{\Lambda} \bar{\eta}^{\Lambda}\right\}\right| V\right\rangle= \\
\varepsilon_{a b c} \varepsilon_{d e f}\left\{2 S_{u}^{a d} \gamma_{5} C S_{d}^{b e} C \gamma_{5} S_{s}^{c f}+2 S_{s}^{a d} \gamma_{5} C S_{d}^{b e} C \gamma_{5} S_{u}^{c f}+\right. \\
2 S_{s}^{a d} \gamma_{5} C S_{u}^{b e} C \gamma_{5} S_{d}^{c f}+2 S_{d}^{a d} \gamma_{5} C S_{u}^{b e} C \gamma_{5} S_{s}^{c f} \\
-\left[S_{u}^{a d} \gamma_{5} C S_{s}^{b e} C \gamma_{5} S_{d}^{c f}+S_{d}^{a d} \gamma_{5} C S_{s}^{b e} C \gamma_{5} S_{u}^{c f}\right]+\ldots
\end{gathered}
$$

and we retain for a moment only these terms to show explicitly the group-theoretical structure. In fact, every three lines as to the quark flavors are of the form

$$
\Lambda=2 u d s+2 s d u+2 s u d+2 d u s-u s d-d s u
$$

in agreement with [3].

Transition magnetic moment "master formula" could be read from [3] as

$$
\begin{gathered}
\sqrt{3} \Sigma^{0} \Lambda \equiv \sqrt{3}\left\langle 0\left|T\left\{\eta^{\Sigma^{0}} \bar{\eta}^{\Lambda}\right\}\right| 0\right\rangle= \\
\varepsilon_{a b c} \varepsilon_{d e f}\left\{\left[S_{u}^{a d} \gamma_{5} C S_{d}^{b e} C \gamma_{5} S_{s}^{c f}+S_{s}^{a d} \gamma_{5} C S_{d}^{b e} C \gamma_{5} S_{u}^{c f}\right]-\right. \\
{\left[S_{s}^{a d} \gamma_{5} C S_{u}^{b e} C \gamma_{5} S_{d}^{c f}+S_{d}^{a d} \gamma_{5} C S_{u}^{b e} C \gamma_{5} S_{s}^{c f}\right]+\ldots} \\
{\left[S_{u}^{a d} \operatorname{Tr}\left[\gamma_{5} C S_{d}^{b e} C \gamma_{5} S_{s}^{c f}\right]+S_{s}^{a d} \operatorname{Tr}\left[\gamma_{5} C S_{d}^{b e} C \gamma_{5} S_{u}^{c f}\right]\right]-} \\
{\left[S_{s}^{a d} \operatorname{Tr}\left[\gamma_{5} C S_{u}^{b e} C \gamma_{5} S_{d}^{c f}\right]+S_{d}^{a d} \operatorname{Tr}\left[\gamma_{5} C S_{u}^{b e} C \gamma_{5} S_{s}^{c f}\right]\right]+\ldots}
\end{gathered}
$$

One can see that every two lines as to the quark flavors are of the form

$$
u d s+s d u-s u d-d u s
$$

Canceling identical terms in the full expression, we obtain

$$
\sqrt{3} \Sigma^{0} \Lambda=\varepsilon_{a b c} \varepsilon_{d e f}\left\{\left[S_{u}^{a d} \gamma_{5} C S_{d}^{b e} C \gamma_{5} S_{s}^{c f}+S_{s}^{a d} \gamma_{5} C S_{d}^{b e} C \gamma_{5} S_{u}^{c f}\right]-\right.
$$




$$
\begin{gathered}
{\left[S_{s}^{a d} \gamma_{5} C S_{u}^{b e} C \gamma_{5} S_{d}^{c f}+S_{d}^{a d} \gamma_{5} C S_{u}^{b e} C \gamma_{5} S_{s}^{c f}\right]+} \\
{\left[S_{u}^{a d} \operatorname{Tr}\left[\gamma_{5} C S_{d}^{b e} C \gamma_{5} S_{s}^{c f}\right]-S_{d}^{a d} \operatorname{Tr}\left[\gamma_{5} C S_{u}^{b e} C \gamma_{5} S_{s}^{c f}\right]\right]+\ldots}
\end{gathered}
$$

New relations (we restrict ourselves with the first terms) yield

$$
\begin{gathered}
\left.\Sigma^{0} \Lambda\right|^{(d \leftrightarrow s)}+\left.\Sigma^{0} \Lambda\right|^{(u \leftrightarrow s)}= \\
\varepsilon_{d e f}\left\{\left[S_{u}^{a d} \gamma_{5} C S_{s}^{b e} C \gamma_{5} S_{d}^{c f}+S_{d}^{a d} \gamma_{5} C S_{s}^{b e} C \gamma_{5} S_{u}^{c f}\right]-\right. \\
\left.\left[S_{d}^{a d} \gamma_{5} C S_{u}^{b e} C \gamma_{5} S_{s}^{c f}+S_{s}^{a d} \gamma_{5} C S_{u}^{b e} C \gamma_{5} S_{d}^{c f}\right]\right\}+ \\
\varepsilon_{a b c} \varepsilon_{d e f}\left\{\left[S_{s}^{a d} \gamma_{5} C S_{d}^{b e} C \gamma_{5} S_{u}^{c f}+S_{u}^{a d} \gamma_{5} C S_{d}^{b e} C \gamma_{5} S_{s}^{c f}\right]-\right. \\
{\left[S_{u}^{a d} \gamma_{5} C S_{s}^{b e} C \gamma_{5} S_{d}^{c f}+S_{d}^{a d} \gamma_{5} C S_{s}^{b e} C \gamma_{5} S_{u}^{c f}\right]=\Sigma^{0} \Lambda .}
\end{gathered}
$$

One can see that every two lines, as to the quark flavors, are of the form

$$
\begin{gathered}
(u s d+d s u-d u s-s u d)+ \\
(s d u+u d s-u s d-d s u)= \\
u d s+s d u-s u d-d u s .
\end{gathered}
$$

Let us give an example. In [5] a QCD SR for the magnetic transition moment was written, which reads in terms of various condensates $a_{q}=-(2 \pi)^{2}\langle\bar{q} q\rangle, b=\left\langle g_{s}^{2} G^{2}\right\rangle$ and so on [5],[6] (for a moment we maintain $a_{u}, a_{d}$ different, the same for other condensates and quark masses $\left.m_{u}, m_{d}\right)$ :

$$
\begin{gathered}
S R\left(\Sigma^{0} \Lambda\right)=\left(e_{u}-e_{d}\right) \frac{M^{6}}{4 L^{4 / 9}}+\frac{L^{4 / 9}}{9 M^{2}}\left[e_{s} a_{s}\left(a_{u}-a_{d}\right)+e_{u} a_{s}\left(a_{u}-3 a_{d}\right)\right. \\
\left.-e_{d} a_{s}\left(a_{d}-3 a_{u}\right)\right]+\frac{M^{2} b}{24 L^{4 / 9}}\left(e_{u}-e_{d}\right) \\
\frac{M^{2} b}{144 L^{4 / 9}}\left[\left[\ln \left(\frac{M^{2}}{\Lambda^{2}}\right)-1-\gamma_{E M}\right]+4\left[\ln \left(\frac{M^{2}}{\Lambda^{2}}\right)-\gamma_{E M}-\frac{M^{2}}{2 \Lambda^{2}}\right]\right]\left(e_{u}-e_{d}\right) \\
+\left[\frac{-\chi}{6 L^{4 / 27}}\left(M^{2}-\frac{m_{(s) 0}^{2}}{8 L^{4 / 9}}\right) \times\left[a_{s}\left(e_{u} \chi_{u} a_{u}-e_{d} \chi_{d} a_{d}\right)+e_{s} \chi_{s} a_{s}\left(a_{u}-a_{d}\right)\right]+\right. \\
\frac{M^{2}}{L^{4 / 9}} \frac{1}{4}\left[2\left(e_{u} a_{u} m_{d}-e_{d} a_{d} m_{u}\right)-2 e_{s} a_{s}\left(m_{u}-m_{d}\right)-\right. \\
\left.+\frac{L^{4 / 9}}{36}(2 \kappa-\xi)\right] \times\left[a_{s}\left(e_{u}\left(2 \kappa_{u}-\xi_{u}\right) a_{u}-e_{d}\left(2 \kappa_{d}-\xi_{d}\right) a_{d}\right)+e_{s}\left(2 \kappa_{s}-\xi_{s}\right) a_{s}\left(a_{u}-a_{d}\right)\right] \\
\left.2 m_{s}\left(e_{d} a_{d}-e_{u} a_{u}\right)+m_{s}\left(e_{u} a_{d}-e_{d} a_{u}\right)+e_{s}\left(a_{d} m_{u}-a_{u} m_{d}\right)\right] \\
-\frac{M^{4}}{4 L^{28 / 27}}\left[\left(e_{u} a_{u} \chi_{u}-e_{d} a_{d} \chi_{d}\right) m_{s}+e_{s} a_{s} \chi_{s}\left(m_{u}-m_{d}\right)\right]+ \\
\frac{1}{18}\left[\left(e_{u} a_{u}\left(2 \kappa_{u}-\xi_{u}\right)-e_{d} a_{d}\left(2 \kappa_{d}-\xi_{d}\right) m_{s}+e_{s} a_{s}\left(2 \kappa_{s}-\xi_{s}\right)\left(m_{u}-m_{d}\right)\right] M^{2}-\right. \\
\frac{M^{2}}{6}\left[\left(e_{u} a_{u} \kappa_{u}-e_{d} a_{d} \kappa_{d}\right) m_{s}+e_{s} a_{s} \kappa_{s}\left(m_{u}-m_{d}\right)\right] \times \\
\left.\times\left[\ln \left(\frac{M^{2}}{\Lambda^{2}}\right)-1-\gamma_{E M}\right]\right]=\beta_{\Sigma} \beta_{\Lambda} \sqrt{3} \mu_{\Sigma^{0} \Lambda} e^{-\bar{m}^{2} / M^{2}}\left(1+A_{\Sigma^{0} \Lambda} M^{2}\right)+\ldots
\end{gathered}
$$

One can see that each term in this formula satisfies our new relation

$$
\left.\langle\Sigma \Lambda\rangle\right|^{(u \leftrightarrow s)}+\left.\langle\Sigma \Lambda\rangle\right|^{(d \leftrightarrow s)}=\langle\Sigma \Lambda\rangle .
$$




\section{Baryon Distribution Amplitudes}

Our group-theoretical relations for the product of interpolating currents do not work if we go to higher $Q^{2}$ (baryon electromagnetic form factors at high momentum transfer, form factors of lepton betadecay of the heavy baryons etc). QCD SR's now are formulated for the product of baryon interpolating current and the photon or weak boson one. In the ket bracket we have now not photon, meson or weak boson, but a baryon. Correspondingly, one should work not with photon or boson distrubution amplitudes, but with the baryon distribution amplitudes [8].

The resulting formulas are even more cumbersome than in the case of QCD SR's based on the product of the baryon interpolating currents, and the final formulas of several authors for the same subject are different. That is why we decided to search for some group theoretical relations also for this case. New group-theoretical relations for the SR's with baryon distribution amplitudes read as

$$
\begin{gathered}
\text { (i) } \sqrt{3}\left\langle 0\left|\eta_{\Lambda} \eta_{\gamma}\right| \Lambda\right\rangle=2\left\langle 0\left|\eta_{\Sigma}^{(u \leftrightarrow s)} \eta_{\gamma}\right| \Lambda\right\rangle+\left\langle 0\left|\eta_{\Sigma} \eta_{\gamma}\right| \Lambda\right\rangle \\
\text { (ii) } \sqrt{3}\left\langle 0\left|\eta_{\Lambda} \eta_{\gamma}\right| \Sigma\right\rangle=2\left\langle 0\left|\eta_{\Sigma}^{(u \leftrightarrow s)} \eta_{\gamma}\right| \Sigma\right\rangle+\left\langle 0\left|\eta_{\Sigma} \eta_{\gamma}\right| \Sigma\right\rangle \\
\text { (iii) } \sqrt{3}\left\langle 0\left|\eta_{\Sigma} \eta_{\gamma}\right| \Lambda\right\rangle=-2\left\langle 0\left|\eta_{\Lambda}^{(d \leftrightarrow s)} \eta_{\gamma}\right| \Lambda\right\rangle+\left\langle 0\left|\eta_{\Lambda} \eta_{\gamma}\right| \Lambda\right\rangle \\
\text { (iv) } \sqrt{3}\left\langle 0\left|\eta_{\Sigma} \eta_{\gamma}\right| \Sigma\right\rangle=-2\left\langle 0\left|\eta_{\Lambda}^{(d \leftrightarrow s)} \eta_{\gamma}\right| \Sigma\right\rangle+\left\langle 0\left|\eta_{\Lambda} \eta_{\gamma}\right| \Sigma\right\rangle
\end{gathered}
$$

We have 4 different matrix elements and try to write the solutions in the NRQM as

$$
\left\langle 0\left|\eta_{\Lambda} \eta_{\gamma}\right| \Lambda\right\rangle=a e_{u}+b e_{d}+c e_{s} .
$$

Putting it into (iii) we get

$$
\left\langle 0\left|\eta_{\Lambda} \eta_{\gamma}\right| \Lambda\right\rangle=a\left(e_{u}-e_{d}\right), \quad \sqrt{3}\left\langle 0\left|\eta_{\Sigma} \eta_{\gamma}\right| \Lambda\right\rangle=-a\left(e_{u}+e_{d}-2 e_{s}\right) .
$$

Similar calculations can be made for relations (ii) and (iv) wherefrom general solution would be

$$
\left\langle 0\left|\eta_{\Sigma} \eta_{\gamma}\right| \Sigma\right\rangle=\tilde{a}\left(e_{u}+e_{d}-2 e_{s}\right), \quad \sqrt{3}\left\langle 0\left|\eta_{\Lambda} \eta_{\gamma}\right| \Sigma\right\rangle=3 \tilde{a}\left(e_{d}-e_{u}\right)
$$

Thus solutions for the matrix elements $\left\langle 0\left|\eta_{\Sigma} \eta_{\gamma}\right| \Sigma\right\rangle$ and $\sqrt{3}\left\langle 0\left|\eta_{\Sigma} \eta_{\gamma}\right| \Lambda\right\rangle$ should be similar up to some overall factor. Surprisingly enough we have seen that these relations are valid for QCD sum rules in $[9,10]$. As an example we take $\Sigma \Lambda$ transition formula from Eq.(9) in [10] (we write only the 1st part of it and put $m_{s}=0$ which does not affect our conclusions)

$$
F_{1}^{\Sigma \Lambda}\left(Q^{2}\right)=\frac{1}{4 \sqrt{2} \lambda_{\Sigma^{0}}} e^{m_{\Sigma^{0}}^{2}} \int_{x_{0}}^{1} d x\left(-\frac{\rho_{2}(x)}{x}+\frac{\rho_{4}(x)}{M^{2} x^{2}}-\frac{\rho_{6}(x)}{2 M^{4} x^{3}}\right) e^{-\left(\frac{Q^{2}(1-x)}{M^{2} x}+\frac{m_{\Lambda}^{2} x}{M^{2}}\right)}+\ldots
$$

where

$$
\begin{gathered}
\rho_{6}(x)=4 m_{\Lambda}^{3} x\left(m_{\Lambda}^{2}+Q^{2}\right)(1+\beta)\left(e_{u} B_{6}(x)+e_{d} \tilde{B}_{6}(x)+2 e_{s} \hat{B}_{6}(x)\right) \\
\rho_{2}(x)=-2 e_{u} m_{\Lambda}\left\{\left[(1-\beta)\left(D_{2}+C_{2}\right)-(1+\beta)\left(B_{2}-B_{4}\right)\right](x)\right. \\
\left.+x \int d x_{3}\left[(1-\beta)\left(A_{3}+2 V_{1}-3 V_{3}\right)-(1+\beta)\left(P_{1}+S_{1}-5 T_{1}+10 T_{3}\right)\right]\left(x, 1-x-x_{3}, x_{3}\right)\right\} \\
+2 e_{d} m_{\Lambda}\left\{\left[(1-\beta)\left(\tilde{D}_{2}-\tilde{C}_{2}\right)+(1+\beta)\left(\tilde{B}_{2}-\tilde{B}_{4}\right)\right](x)\right. \\
\left.+x \int d x_{1}\left[(1-\beta)\left(A_{3}-2 V_{1}+3 V_{3}\right)-(1+\beta)\left(P_{1}+S_{1}+5 T_{1}-10 T_{3}\right)\right]\left(x_{1}, x, 1-x_{1}-x\right)\right\}
\end{gathered}
$$




$$
\begin{gathered}
+4 e_{s}\left\{m_{\Lambda}\left[(1-\beta) \hat{C}_{2}-(1+\beta)\left(\hat{B}_{2}-B_{4}\right)\right](x)\right. \\
+\int d x_{1}\left\{(1-\beta)\left(m_{\Lambda} x V_{3}+(1+\beta) m_{\Lambda} x\left(2 T_{3}-T_{1}\right]\right\}\left(x_{1}, 1-x_{1}-x, x\right)\right\}
\end{gathered}
$$

and we omitted $\rho_{4}$ as it is too lengthy. All the $A, B, C$, etc., are defined in [10].

If we change all the $\Lambda$ symbols to $\Sigma^{0}$ ones we just arrive at the formulas for $F_{1}^{\Sigma^{0}}\left(Q^{2}\right)$ for the $\Sigma^{0}$ form factor of [9] (see the Appendix of that reference):

$$
F_{1}^{\Sigma^{0}}\left(Q^{2}\right)=\frac{1}{4 \sqrt{2} \lambda_{\Sigma^{0}}} e^{m_{\Sigma}^{2}} \int_{x_{0}}^{1} d x\left(-\frac{\rho_{2}(x)}{x}+\frac{\rho_{4}(x)}{M^{2} x^{2}}-\frac{\rho_{6}(x)}{2 M^{4} x^{3}}\right) e^{-\left(\frac{Q^{2}(1-x)}{M^{2} x}+\frac{m_{\Sigma}^{2} x}{M^{2}}\right)}+\ldots
$$

where $\rho_{2,4,6}$ are the same as in (10) with the only change of $m_{\Lambda}$ to $m_{\Sigma}$, which proves the validity of our relation for the baryon DA's.

\section{Conclusions}

1. We have obtained the new group-theoretical relation for the QCD SR and proved it for general "master formulas" as well as for the QCD SR's for $\Sigma \Lambda$ magnetic transition moment in terms of various condensates. This relation is valid also for the QCD sum rules on the light cone.

2. We have established new relations for the case of baryon DA's and have shown its validity on the example of the LC QCD SR for the electromagnetic form factors of $\Sigma$ baryon and $\Sigma-\Lambda$ transition.

3. These relations are not constraint to the octet representation of SU(3) but also can be written for sextet and antitriplet that is for $\Sigma_{Q}$ and $\Lambda_{Q}$ and for pairs of heavy cascade hyperons $\Xi_{Q}$ and $\Xi_{Q}^{\prime}$ with $Q=c, b$ with the appropriate changes.

We thank the Organizers of the Workshop QCD 2014 for their kind hospitality.

\section{References}

[1] M.A. Shifman, V.I. Vainshtein, and V.I. Zakharov, Nucl. Phys. B147, 385 (1979)

[2] B.L. Ioffe, Nucl. Phys B188, 317 (1981)

[3] A. Ozpineci, S. Yakovlev, and V. Zamiralov, At. Nucl. Phys. 68, 304 (2005); Mod. Rev. Lett. 20, 243 (2005)

[4] Lai Wang and Frank X. Lee, Phys. Rev. D78, 013003 (2008)

[5] Shi-lin Zhu, W.-Y.P. Hwang, and Ze-sen Yang, Phys. Rev. D57, 1524 (1998)

[6] Charles B. Chiu, J. Pasupathy, and S.J. Wilson, Phys. Rev. D33, 1961 (1986)

[7] T. Aliev, A. Ozpineci, and M. Savci, Phys. Rev. D66, 016002 (2002)

[8] P. Ball and V. M. Braun, Phys. Rev. D55, 5561 (1997); Phys. Rev. D58, 094016 (1998)

[9] T.M. Aliev, K. Azizi, and M. Savci, arXiv:1303.6798 [hep-ph]

[10] T.M. Aliev, K. Azizi, and M. Savci, arXiv:1303.6897v2[hep-ph] 
